\title{
SESSIS-TRIGGERED TAKOTSUBO CARDIOMYOPATHTY: A SEVERE CASE RESCUED BY VENO-ATRIAL EXTRACORPOREAL MEMBRANE OXYGENATION
}

\author{
Kazuya Omura ${ }^{1}$, Tetsuya Hoshino ${ }^{1}$ \\ ${ }^{1}$ Saiseikai Yokohamashi Tobu Hospital, Department of Critical Care Medicine, Yokohama, Japan
}

Cite this article as:

\section{Abstract}

Introduction: The use of extracorporeal membrane oxygenation (ECMO) for sepsis-related shock remains controversial. We report a shock case with sepsis-triggered Takotsubo cardiomyopathy, who was successfully rescued using veno-atrial ECMO.

Case Report: A 76-year-old woman with hypoglycemia and a high fever was diagnosed as sepsis-triggered Takotsubo cardiomyopathy. She subsequently developed severe shock. Veno-atrial ECMO was initiated, then the patient responded quickly and recovered fully. She was later diagnosed as having had cardiogenic shock, based on her clinical course.

Conclusion: Although distinguishing cardiogenic from septic shock is difficult, the initiation of veno-atrial ECMO in severe cases of sepsis-triggered Takotsubo cardiomyopathy might be reasonable.

Keywords: Takotsubo Cardiomyopathy, Shock, Sepsis, Extracorporeal membrane oxygenation, cardiogenic shock

Received:08.02.2018 Accepted:08.02.2018

\section{Introduction}

Takotsubo cardiomyopathy is a reversible disease characterized by a dysfunction of the mid to apical segments of the left ventricle and apical ballooning. This condition is generally known to be relatively benign; however, several cases of serious complications, such as cardiogenic shock or fatal arrhythmia have been reported. In severe cases of cariogenic shock with progressive organ dysfunction, the initiation of intraaortic balloon pumping (IABP) and/or extracorporeal membrane oxygenation(ECMO) can be considered.

Takotsubo cardiomyopathy can be divided into primary and secondary disease. Sepsis is one of the major triggers of secondary Takotsubo cardiomyopathy. In severe cases of sepsis-triggered Takotsubo cardiomyopathy, septic shock and cardiogenic shock can be difficult to distinguish. Moreover, the use of ECMO for both septic shock and cardiogenic shock in secondary Takotsubo cardiomyopathy remains controversial. When a patient develops severe shock with organ failure, however, a decision as to whether to initiate ECMO must be made.

We report a patient with sepsis-triggered Takotsubo cardiomyopathy and severe shock who was successfully rescued using veno-atrial ECMO. 


\section{Case Reports}

A 76-year-old woman with no past medical history presented in the emergency department with an altered mental status. She was comatose upon admission, with a Glasgow Coma Scale of 3 (E1V1M1). A laboratory examination revealed a low blood glucose level of $14 \mathrm{mg} / \mathrm{dL}$. After glucose administration, her mental status recovered immediately and fully. Her creatine kinase (CK) and CK-MB levels (63 mg/dL and $9 \mathrm{mg} / \mathrm{dL}$, respectively) were within the normal ranges. Her troponin- $T$ level $(0.09 \mathrm{ng} / \mathrm{dL})$ was slightly elevated. An electrocardiogram showed poor R progression in V1-3 and a slight ST elevation in V4-6. An echocardiogram showed a decreased left ventricular ejection fraction with asynergy (hypokinesia on the anterior wall). She was admitted to the general ward under a suspicion of having acute coronary syndrome.

Four hours after arrival, her CK/CK-MB and troponin-T levels became elevated at $203 \mathrm{U} / \mathrm{L}, 27 \mathrm{U} / \mathrm{L}$ and $1.27 \mathrm{ng} / \mathrm{dL}$, respectively. An emergency coronary angiography was performed, revealing normal coronary arteries. A left ventriculogram revealed basal hyperkinesia and apical ballooning, and she was diagnosed as having Takotsubo cardiomyopathy (Fig. 1). During the coronary angiography, her hemodynamic status deteriorated critically. She was admitted to the intensive care unit (ICU) and treated with the continuous administration of norepinephrine and the initiation of IABP.

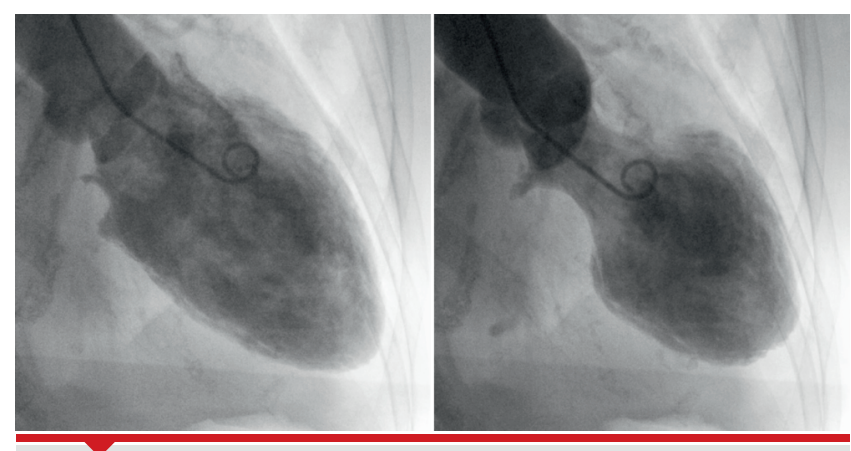

FIGURE 1. Left ventriculogram a left ventriculogram revealed basal hyperkinesia and apical ballooning.

At the time of admission to the ICU, her body temperature was elevated $\left(40^{\circ} \mathbf{C}\right)$ and she was hypotensive. She developed shock with multiorgan failure; her SOFA (Sequential Organ Failure Assessment) score at admission was 13. A laboratory examination showed an elevated procalcitonin level (1.93 $\mathrm{ng} / \mathrm{mL}$ ). Because of her persistent hypoglycemia and elevated procalcitonin and lactate levels, septic shock was suspected. Endotracheal intubation was performed, and the administration of an antidiuretic hormone and a steroid was initiated to maintain her blood pressure. Meropenem was empirically started. Because of her hemodynamic instability, veno-atrial ECMO was initiated 14 hours after her admission to the ICU (Figure 2).

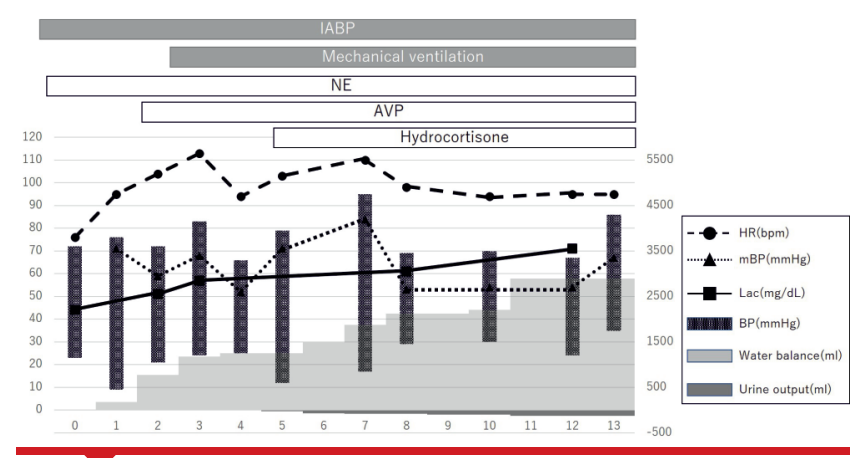

FIGURE 2.

She responded quickly to the veno-atrial ECMO, enabling the administration of the vasopressor agents to be stopped. Haemophilus influenzae was detected in a sputum culture taken at the time of hospitalization, but no bacterial species were found in her blood; consequently, her antibiotics prescription was changed to ceftriaxone on the 3rd day after the initiation of veno-atrial ECMO. Her cardiac function recovered along with an improvement in her inflammatory response, and veno-atrial ECMO was discontinued on the 8th day (Table 1). She was transferred to a rehabilitation hospital without any complications on the 34th day.

TABLE 1. Laboratory and echocardiography findings.

\begin{tabular}{|r|l|c|c|c|c|c|c|c|}
\hline & & before & day1 & day2 & day4 & day6 & day8 & da10 \\
\hline WBC & $\left(10^{3} / \mathrm{mm}^{3}\right)$ & 6.69 & 28.64 & 21.52 & 13.74 & 8.94 & 18.14 & 19.47 \\
\hline CRP & $(\mathrm{mg} / \mathrm{dL})$ & 4.83 & 8.07 & 5.36 & 2.68 & 3.4 & 6.59 & 2.88 \\
\hline CK & $(\mathrm{U} / \mathrm{L})$ & 410 & 1163 & 1177 & 469 & 279 & 162 & 34 \\
\hline EF & $(\%)$ & - & - & 26.6 & - & 49.9 & - & 72.2 \\
\hline
\end{tabular}

CK: creatine kinase, CRP: C-reactive protein, EF: ejection fraction, WBC: white blood cell

\section{Discussion}

We identified two important clinical issues. When a patient with sepsis-triggered Takotsubo cardiomyopathy develops shock, it is difficult to distinguish cardiogenic shock from septic shock. In such situations, veno-atrial ECMO can be a useful treatment option.

First, cardiogenic shock is difficult to distinguish from septic shock in patients with sepsis-triggered Takotsubo cardiomyopathy. Although the procalcitonin level is recognized as a specific marker of infection, an elevated procalcitonin level of greater than $2 \mathrm{ng} / \mathrm{mL}$ is frequently observed in cardiogenic shock patients with multiorgan failure. A cutoff concentration of $10 \mathrm{ng} / \mathrm{mL}$ is reportedly appropriate for the diagnosis of a complicating systemic infection (1). In patients with Takotsubo cardiomyopathy and a background of infectious disease, the procalcitonin level is likely to be relatively high; therefore, it might not be useful for distinguishing cardiogenic shock from septic shock. 
Because few reports describing sepsis-triggered Takotsubo cardiomyopathy are available, the clinical manifestations of severe cases remain relatively unknown. A systematic review of sepsis triggered-Takotsubo cardiomyopathy identified 23 papers reporting a total of 26 patients including 3 patients with shock (2). One was diagnosed as having cardiogenic shock based on findings obtained using a pulmonary artery catheter, but the other two were diagnosed based on their clinical course. In the present case, the patient was diagnosed as having cardiogenic shock, rather than septic shock, based on her clinical course, since her hemodynamic status improved immediately after the initiation of veno-atrial ECMO.

Second, veno-atrial ECMO can be a treatment option for cardiogenic shock in patients with sepsis-triggered Takotsubo cardiomyopathy. If a patient with primary Takotsubo cardiomyopathy develops cardiogenic shock and progressive multiorgan failure, the advice of a specialist regarding the use of mechanical devices, including ECMO, is recommended (35). On the other hand, there are no established recommendations for the use of ECMO in patients with secondary Takotsubo cardiomyopathy. In severe cases with progressive multiorgan dysfunction, the nature, severity, and prognosis of the underlying medical condition must be considered when determining whether to initiate mechanical support (6). The initiation of ECMO should be performed with caution because its use for the treatment of septic shock remains controversial (7). In the current case, the patient's hemodynamics status improved quickly after the initiation of ECMO. In patients with sepsis-triggered Takotsubo cardiomyopathy who develop shock, a favorable prognosis can be expected using ECMO if the shock is cardiogenic.

The number of sepsis cases is increasing (8), resulting in more opportunities to treat sepsis patients. Sepsis-induced myocardial dysfunction is widely known as septic cardiomyopathy and sepsis-triggered Takotsubo cardiomyopathy; the incidence is between 40\%-60\% for diagnoses within the first 3 days (9). Recently, septic cardiomyopathy and sepsis-triggered Takotsubo cardiomyopathy were found to have different mechanisms. However, such distinctions are unlikely to have been made in past reports. It is very important to examine cardiac function in sepsis patients. The use of catecholamine is recommended for septic cardiomyopathy, whereas the avoidance or withdrawal of exogenous catecholamine is recommended for Takotsubo cardiomyopathy. Distinguishing between septic cardiomyopathy and Takotsubo cardiomyopathy is especially important in cases with left ventricular dysfunction. If the diagnosis is Takotsubo cardiomyopathy, a favorable prognosis can be expected; thus, physicians may not hesitate to initiate aggressive treatment, including ECMO.

\section{Conclusion}

When patients with sepsis-triggered Takotsubo cardiomyopathy develop shock, distinguishing cardiogenic shock from septic shock can be difficult. In this situation, the initiation of veno-atrial ECMO might be reasonable. Physicians must be aware that in addition to the procalcitonin level, a comprehensive diagnosis based on the clinical course is necessary to distinguish between cardiogenic shock and septic shock. Even in cases with severe shock, if the cause is cardiogenic shock arising from Takotsubo cardiomyopathy, ECMO can be considered as a reasonable treatment option. Previous reports may have contained unrecognized cases of Takotsubo cardiomyopathy, and the accumulation of further reports is needed to clarify the manifestations of severe cases of sepsis-triggered Takotsubo cardiomyopathy.

Informed Consent: The case report has written in an anonymous characteristic, thus secret and detailed data about the patient has removed. Editor and reviewers can know and see these detailed data. These data are backed up by editor and by reviewers.

Peer-review: none

Iconflict of Interest: none

Financial Disclosure: none

\section{Reference:}

1. Geppert A, Steiner A, Delle-Karth G, Heinz G, Huber K. Usefulness of procalcitonin for diagnosing complicating sepsis in patients with cardiogenic shock. Intensive Care Med. 29:1384-1389, 2003.

2. Cappelletti S, Ciallella C, Aromatario M, Ashrafian H, Harding S, Athanasiou T. Takotsubo Cardiomyopathy and Sepsis. Angiology. 68:288303, 2017.

3. Bonacchi M, Maiani M, Harmelin G, Sani G. Intractable cardiogenic shock in stress cardiomyopathy with left ventricular outflow tract obstruction: is extra-corporeal life support the best treatment? Eur J Heart Fail 11:721-727, 2009.

4. Lazzeri C, Sori A, Bernardo P, Picariello C, Gensini GF, Valente S. Inhospital refractory cardiac arrest treated with extracorporeal membrane oxygenation: a tertiary single center experience. Acute Card Care 15:47-51, 2013

5. Donker DW, Pragt E, Weerwind PW, Holtkamp JW, Vainer J, Mochtar B, et al. Rescue extracorporeal life support as a bridge to reflection in fulminant stress-induced cardiomyopathy. Int J Cardiol 154:e54-e56, 2012.

6. Lyon AR, Bossone E, Schneider B, Sechtem U, Citro R, Underwood SR, et al. Current state of knowledge on Takotsubo syndrome: a Position Statement from the Taskforce on Takotsubo Syndrome of the Heart Failure Association of the European Society of Cardiology. European Journal of Heart Failure doi:10.1002/ejhf.424, 2015.

7. Combes A. Role of VA ECMO in septic shock: Does it work? Qatar Med J. 4th Annual ELSO-SWAC Conference 2017: DOI: 10.5339/qmj.2017. swacelso.24, 2017.

8. Cohen J, Vincent JL, Adhikari NK, Machado FR, Angus DC, Calandra T, et al. Sepsis: a roadmap for future research. Lancet Infect Dis 15:581-614, 2015. 9. Vieillard-Baron A, Caille V, Charron C, Belliard G, Page B, Jardin F. Actual incidence of global left ventricular hypokinesia in adult septic shock. Crit Care Med 36:1701-1706, 2008. 